\title{
Intracisternal Application of Endotoxin Enhances the Susceptibility to Subsequent Hypoxic-Ischemic Brain Damage in Neonatal Rats
}

\author{
AUDREY B.C. COUMANS, JOHANNES MIDDELANIS, YVES GARNIER, \\ HANS-MARTIN VAIHINGER, STEPHEN L. LEIB, MONIKA U. VON DUERING, \\ TOM H.M. HASAART, ARNE JENSEN, AND RICHARD BERGER
}

\begin{abstract}
Department of Obstetrics and Gynecology [A.B.C.C., T.H.M.H.], University Hospital Maastricht, The
Netherlands; Department of Obstetrics and Gynecology [J.M., Y.G., H.-M.V., A.J., R.B.], University of

Bochum, Germany; Institute for Infectious Diseases [S.L.L.], University of Bern, Switzerland; and Institute

for Neuroanatomy [M.U.V.D.], University of Bochum, Germany
\end{abstract}

\begin{abstract}
ABS
Perinatal brain damage is associated not only with hypoxic-
ischemic insults but also with intrauterine inflammation. A com-
bination of antenatal inflammation and asphyxia increases the
risk of cerebral palsy $>70$ times. The aim of the present study
was to determine the effect of intracisternal (i.c.) administration
of endotoxin [lipopolysaccharides (LPS)] on subsequent hy-
poxic-ischemic brain damage in neonatal rats. Seven-day-old
Wistar rats were subjected to i.c. application of NaCl or LPS (5
$\mu$ g/pup). One hour later, the left common carotid artery was
exposed through a midline neck incision and ligated with 6-0
surgical silk. After another hour of recovery, the pups were
subjected to a hypoxic gas mixture (8\% oxygen/92\% nitrogen)
for 60 min. The animals were randomized to four experimental
groups: 1$)$ sham control group, left common carotid artery
exposed but not ligated $(n=5) ; 2)$ LPS group, subjected to i.c.
application of LPS ( $n=7) ; 3$ ) hypoxic-ischemic study group, i.c.
injection of NaCl and exposure to hypoxia after ligation of the
left carotid artery ( $n=17)$; or 4$)$ hypoxic-ischemic/LPS study
group, i.c. injection of LPS and exposure to hypoxia after ligation
\end{abstract}
Hypoxic-ischemic cerebral damage is an important contributor to perinatal mortality and morbidity, including long-term neurologic sequelae in term and preterm fetuses $(1,2)$. However, as shown in recent studies, perinatal brain injury may be associated not only with hypoxic-ischemic insults but also with an ascending intrauterine inflammation before or during birth (3). It is widely known that Gram-negative anaerobic bacteria are involved in colonization and infection of the genitourinary

Received March 27, 2002; accepted October 10, 2002.

Correspondence: Tom H.M. Hasaart, M.D., Ph.D., Department of Obstetrics and Gynecology, University Hospital Maastricht, P.O. BOX 5800, 6202 AZ Maastricht, The Netherlands; e-mail: thas@sgyn.azm.nl

This work was supported by Deutsche Forschungsgemeinschaft (Be 1688/4-3), The Dutch Foundations "De Drie Lichten," and "De Oranjekliniek" as well as the Swiss National Science Foundation (32-61654.00).

DOI: 10.1203/01.PDR.0000059221.40073.82 of the left carotid artery $(n=19)$. Seven days later, neonatal brains were assessed for neuronal cell damage. In a second set of experiments, rat pups received an i.c. injection of LPS ( $5 \mu \mathrm{g} / \mathrm{pup})$ and were evaluated for tumor necrosis factor- $\alpha$ expression by immunohistochemistry. Neuronal cell damage could not be observed in the sham control or in the LPS group. In the hypoxicischemic/LPS group, neuronal injury in the cerebral cortex was significantly higher than in animals that were subjected to hypoxia/ischemia after i.c. application of $\mathrm{NaCl}$. Injecting LPS intracisternally caused a marked expression of tumor necrosis factor- $\alpha$ in the leptomeninges. Applying LPS intracisternally sensitizes the immature rat brain to a subsequent hypoxicischemic insult. (Pediatr Res 53: 770-775, 2003)

Abbreviations
LPS, lipopolysaccharides
TNF- $\boldsymbol{\alpha}$, tumor necrosis factor- $\alpha$
i.c., intracisternal
TLR, toll-like receptor

tract in pregnant women, which could affect labor and preterm birth. Because pregnant women with fever and bacteriuria give birth to infants with a higher incidence of neurologic defects at $1 \mathrm{y}$ of age than do mothers who are free of urinary tract infection (4), it seems that human maternal endotoxemia is associated with fetal CNS damage. Furthermore, there is a growing body of evidence from epidemiologic studies that exposure to placental inflammation before or during birth is accompanied by cerebral white matter damage, especially in the very preterm fetuses, i.e. those born before $32 \mathrm{wk}$ of gestation (3). In addition, the incidence of peri/intraventricular hemorrhage is significantly increased in premature infants who are exposed to intrauterine inflammation $(5,6)$.

An increasing number of experimental studies showing various effects of endotoxins on cerebral tissue are being pub- 
lished. Thus, administration of endotoxin has been reported to induce an increased cytokine expression in adult rat brains (7-9). In particular, a rise in the release of tumor necrosis factor- $\alpha$ (TNF- $\alpha)$ has been thought to be associated with neuronal cell damage (10). In addition, an increased expression of IL- $1 \beta$ and TNF- $\alpha$ mRNA has been observed in the brain of fetal rats after intraperitoneal application of lipopolysaccharides (LPS) to the dam. This was followed by minimal cerebral injury (11). Similar observations have been described in immature rabbits after uterine infection with bacteria (12) and in newborn kittens after intraperitoneal injection of LPS (13). Only recently, it was reported that intraperitoneal injection of LPS in neonatal rats aggravates hypoxic-ischemic damage in the immature brain (14). To elucidate the effects of endotoxins on fetal cardiovascular control we applied i.v. LPS to preterm fetal lambs (15). During a short period of superimposed asphyxia, these fetuses were not able to maintain circulatory centralization, i.e. to increase blood flow to the central organs at the expense of the peripheral perfusion. In the immediate recovery period, oxygen delivery to the fetal brain was nearly arrested. It is conceivable that under such conditions, fetal brain injury is brought about by hypoxic-ischemic insults.

Under clinical conditions, intrauterine inflammation is often accompanied by fetal asphyxia. The combination of exposure to inflammation and asphyxia during birth has been linked to a dramatic increase in the risk of spastic cerebral palsy (16), suggesting that there may be an interaction between the pathophysiological mechanisms induced by inflammation and perinatal hypoxia. Up to now, few data have been presented on the effect of an early administration of endotoxins before a hypoxic-ischemic insult in the immature brain. In the present study, we therefore investigated whether intracisternal (i.c.) application of LPS enhances the susceptibility to subsequent hypoxic-ischemic brain damage in neonatal rats.

\section{METHODS}

Animals. Experiments were performed on 7-d-old neonatal Wistar rats $(n=61)$ of both sexes. Until the day of the surgery, the pups stayed under controlled conditions in cages with their dams. A constant temperature of $20^{\circ} \mathrm{C}$, a 12 -h day/night rhythm, and food and drink at libitum were maintained. Mean body weight on the surgery day was $14.9 \pm 0.7 \mathrm{~g}$.

Surgical procedure. The pups were held and fixated by hand. After the skin was disinfected with $70 \%$ alcohol, $\mathrm{NaCl}$ or LPS (5 $\mu \mathrm{g} /$ pup; Escherichia coli, O127:B8; Sigma Chemical Co. Aldrich) was manually injected into the cisterna magna as described previously (17). Briefly, a syringe connected to a 32-gauge needle was used to inject $10 \mu \mathrm{L}$ of fluid intracisternally. The landmarks for the injection were the opistion and the crista occipitalis externa on the superior side and the basion on the inferior side. The needle was inserted within the quadrangle bounded by these points approximately one third of the way between the opsition and the basion. To check that the LPS was indeed being injected into the cistern magna, we performed a pilot study on 50 7-d-old rat pups. In these animals, Chinese ink was co-injected with $\mathrm{NaCl}$ or LPS and the correct admin- istration was verified macroscopically. After the first 10 animals, this pilot study had a success rate of $96 \%$.

One hour after i.c. application of LPS, the rat pups were anesthetized deeply by halothane inhalation. The left common carotid artery was exposed through a midline neck incision and ligated with 6-0 surgical silk. The duration of anesthesia and surgery never exceeded $15 \mathrm{~min}$. After surgery, the pups were allowed to recover with their dams for another hour. The pups were placed in an incubator (Intensive Care Incubator 7510, Fa. Dräger, Lübeck, Germany) and were exposed to a hypoxic gas mixture ( $8 \%$ oxygen $/ 92 \%$ nitrogen). The ambient temperature was maintained strictly at $36^{\circ} \mathrm{C}$. After $60 \mathrm{~min}$ of hypoxia, the incubator was opened to room air and the pups returned to their dams (18). In the present study, we did not measure body temperature in the rat pups, because this is extremely difficult to do in such small animals. However, in experiments on adult animals, fever has not been reported after intracerebroventricular administration of LPS $(19,20)$. The experimental protocols were approved by the appropriate institutional review committee and met the guidelines of the governmental agency responsible.

Experimental groups. The pups were randomized to four experimental groups: 1) sham control group: animals of this group underwent the surgical procedure described above except for the ligation of the carotid artery $(n=5) ; 2)$ LPS group: these animals were subjected only to i.c. application of LPS ( $n$ = 7); 3) hypoxic-ischemic study group: Animals received an i.c. injection of $\mathrm{NaCl}$ and were exposed to hypoxia for $60 \mathrm{~min}$ after ligation of the left carotid artery $(n=17) ; 4)$ hypoxicischemic/LPS study group: animals were received an i.c. injection of LPS and were exposed to hypoxia for $60 \mathrm{~min}$ after ligation of the left carotid artery $(n=19)$.

Histology. Seven days after the insult, the rat pups were anesthetized with i.p. thiopental (12 mg/animal), a butterfly needle (21 gauge) was inserted into the left ventricle, and the right atrium was incised. Each animal was perfusion-fixed with $4 \%$ formaldehyde. After perfusion, the brains were removed and stored in the same fixative for at least $3 \mathrm{~d}$. Coronal blocks of the brains were processed in graded ethanols and rotihistol. After embedding in paraffin wax, 5- $\mu$ m-thick coronal slices were sectioned every $500 \mu \mathrm{m}$ and stained with cresyl-violet/fuchsin.

Hypoxic-ischemic brain injury was assessed directly after the brains were removed from the cranial cavity using a macroscopic four-point scale [modified after (21)]: $0=$ no macroscopically detectable damage; $1=$ discrete, delineated, superficial pigmentation of the ipsilateral hemisphere; $2=$ moderate cystic infarction of the ipsilateral hemisphere $(\leq 4$ $\mathrm{mm}) ; 3=$ severe cystic infarction of the ipsilateral hemisphere $(>4 \mathrm{~mm})$.

Hypoxic-ischemic neuronal cell damage within the cerebral cortex was also assessed microscopically. The cortex ipsilateral to the ligated carotid artery was evaluated at six coronal levels (longitudinal coordinates relative to Bregma: A $1.0 \mathrm{~mm}$, A $2.0 \mathrm{~mm}$, A $3.0 \mathrm{~mm}$, A $4.0 \mathrm{~mm}$, A $5.0 \mathrm{~mm}$, A $6.0 \mathrm{~mm}$ ). Coronal level A $2.0 \mathrm{~mm}$ showing the middle of the dorsal hippocampus was taken as the reference plane according to a stereotaxic atlas of 14-d-old rat brains (22). The longitudinal 
coordinates of the other coronal levels were determined by counting the number of the 5 - $\mu \mathrm{m}$-thick histologic slices in posterior and anterior direction. The ipsilateral cortex was analyzed by visual field at a magnification of $250 \times$. Neurons with ischemic cell damage were identified according to the criteria of Brown and Brierley (23). Neuronal cell damage in each microscopic visual field was quantified by the following score: $0-5 \%$ damage (score 1), 5-50\% damage (score 2), $50-95 \%$ damage (score 3), 95-99\% damage (score 4), and $100 \%$ damage (score 5) (24). The histologic score of each coronal level was calculated by averaging the scores of all visual fields analyzed in that level. In the same way, neuronal cell damage of four hippocampal structures (dentate gyrus, CA 4, CA 3, CA 1 and 2) was evaluated at the coronal plane A 2.0 $\mathrm{mm}$ and that of the striatum at the coronal planes A $3.0 \mathrm{~mm}$ and A $4.0 \mathrm{~mm}$. The scoring system used in the present study has been previously established and evaluated by our group. The intraobserver and interobserver reliabilities were assessed as coefficients of variation and amounted to 3 and $10 \%$, respectively. This system has been used by our group and others successfully in a large number of studies (24-26).

In addition, coronal sections were scanned at $2700 \times 2700$ dpi with a film scanner (SprintScan 35 Plus, Polaroid Europe Ltd., Uxbridge, Middlesex, UK), and the digitized images were analyzed using the public domain NIH Image program (Version 1.61, U.S. National Institutes of Health, Bethesda, MD, U.S.A.). Neuronal injury to the cortex was defined as areas of decreased neuronal density, confirmed by simultaneous bright field microscopy, and the area of cortical brain damage was expressed as percentage of the total cortex in each section. The mean value per brain was calculated and used for statistical evaluation (27). All observers involved in the histologic evaluations were blind to the treatment procedures.

Immunohistochemistry. To clarify whether the injected LPS is really active in the immature brain, we studied the expression of TNF- $\alpha$. For this purpose, another group of rat pups ( $n$ $=9$ ) were treated with LPS as described above. At 4, 10, and $24 \mathrm{~h}$ after the application, these animals as well as NaCl-treated control pups $(n=4)$ were killed and perfusion-fixed with $4 \%$ formaldehyde. After incubation for $48 \mathrm{~h}$ in formaldehyde, the brains were transferred into $18 \%$ sucrose. Twenty-microgramthick coronal frozen sections of the brains were processed on a cryostat. For detection of TNF- $\alpha$, anti-rat TNF- $\alpha$ was used as primary antibody (Endogen, Woburn, MA U.S.A.) at a working dilution of 1:1000. After incubation with primary antibody at room temperature for $2 \mathrm{~h}$, the avidin-biotin-horseradish peroxidase system (ABC kit from Vector Laboratories, Burlingame, CA, U.S.A.) was used for detection of immunopositive cells following the manufacturer's instructions. Negative controls were performed by omitting the primary antibody and by preadsorption of the TNF- $\alpha$ antibody with the homologous antigen (PeproTech EC Ltd, London, UK).

Statistics. All data are given as means \pm SD. Statistical differences between groups were evaluated by the $\chi^{2}$ test, Mann-Whitney $U$ test, or unpaired $t$ test when appropriate. $P<$ 0.05 was considered significant.

\section{RESULTS}

Neuronal cell damage could not be observed in sham control animals or in rat pups that were subjected solely to LPS injection into the cisterna magna. In the cerebral cortex of the animals allotted to macroscopic score 1 and 2, only selective neuronal necrosis could be detected without any evidence of infarction. In the neonates that underwent hypoxia-ischemia plus LPS injection, the macroscopically assessed brain injury was significantly higher than that observed in pups that were subjected to hypoxia-ischemia and administration of $\mathrm{NaCl}$ (Table 1). Whereas seven animals of the LPS group (group 4) developed severe cystic cerebral infarction, only mild to moderate damage could be observed in the rat pups of the $\mathrm{NaCl}$ group (group 3). The extent of microscopically determined neuronal cell damage in these two groups is illustrated in Table 2 and Figure 1. Neuronal cell damage was most pronounced in the posterior levels of the cerebral cortex. Except at the levels A 1.0 and A 3.0, neuronal cell injury was significantly higher in the cortex of the animals that were subjected to hypoxiaischemia and LPS injection. No differences between groups could be detected in the hippocampus and striatum. Cellular damage in the white matter could not be observed in any of the brains evaluated. The mean neuronal injury in the cerebral cortex of the animals of experimental group 4 analyzed by the above described CCD-based image analysis system was significantly higher than that in the rat pups of group 3 (8.9 \pm $3.2 \%$ versus $1.2 \pm 0.2 \% ; p<0.001$; Fig. 2 ). In group 4 animals, neuronal cell damage or white matter injury could not be detected in the contralateral hemisphere. We therefore did not randomize rat pups to sham surgery and LPS application.

Ten hours after injection of LPS into the cisterna magna, a weak expression of TNF- $\alpha$ could be observed in the leptomeninges $(n=3)$. After $24 \mathrm{~h}$, this expression was considerably stronger $(n=3$; Fig. 3 ). Most of the cells that were positive for TNF- $\alpha$ staining seemed to be macrophages. No staining for TNF- $\alpha$ could be detected in the cerebral parenchyme at any time after LPS injection. Animals that received an injection of $\mathrm{NaCl}$ also gave no positive staining for TNF- $\alpha(n=4$; Fig. 3$)$.

\section{DISCUSSION}

The central finding of the present study was that endotoxin enhances the susceptibility to subsequent hypoxic-ischemic

Table 1. Macroscopically evaluated hypoxic-ischemic brain injury in neonatal rats

\begin{tabular}{ccc}
\hline Score & $\mathrm{NaCl} / \mathrm{HI}$ & LPS/HI \\
\hline 0 & 8 & 4 \\
1 & 6 & 8 \\
2 & 3 & 0 \\
3 & 0 & $7^{*}$ \\
\hline
\end{tabular}

Rat pups were subjected to either i.c. application of $\mathrm{NaCl}(n=17)$ or LPS $(n=19)$. One hour later, the animals underwent unilateral carotid artery occlusion followed by $60 \mathrm{~min}$ of hypoxia (HI). $0=$ no macroscopically detectable damage; 1 = discrete, delineated, superficial pigmentation of the ipsilateral hemisphere; 2 = moderate cystic infarction of the ipsilateral hemisphere $(\leq 4 \mathrm{~mm}) ; 3=$ severe cystic infarction of the ipsilateral hemisphere $(>4$ $\mathrm{mm})$. Statistically significant differences between control and study group were evaluated by $\chi^{2}$ test $(* p<0.01)$. 
Table 2. Microscopically evaluated hypoxic-ischemic brain injury

\begin{tabular}{ccc}
\multicolumn{3}{c}{ in neonatal rats } \\
\hline & $\mathrm{NaCl} / \mathrm{HI}$ & LPS/HI \\
\hline Cerebral cortex & & \\
Level 1 (A 1.0) & $1.31 \pm 0.21$ & $1.95 \pm 1.25$ \\
Level 2 (A 2.0) & $1.29 \pm 0.23$ & $2.13 \pm 1.20^{*}$ \\
Level 3 (A 3.0) & $1.24 \pm 0.15$ & $1.88 \pm 1.12$ \\
Level 4 (A 4.0) & $1.08 \pm 0.11$ & $1.60 \pm 0.98^{* *}$ \\
Level 5 (A 5.0) & $1.03 \pm 0.10$ & $1.37 \pm 0.90^{* *}$ \\
Level 6 (A 6.0) & $1.03 \pm 0.13$ & $1.39 \pm 1.06^{*}$ \\
Total & $1.18 \pm 0.12$ & $1.81 \pm 1.05^{*}$ \\
Hippocampus & & \\
DG & $1.59 \pm 1.33$ & $1.26 \pm 0.73$ \\
CA 1/2 & $1.59 \pm 1.37$ & $1.11 \pm 0.32$ \\
CA 3 & $1.82 \pm 1.55$ & $1.54 \pm 1.01$ \\
CA 4 & $1.65 \pm 1.32$ & $1.54 \pm 1.20$ \\
Striatum & $1.27 \pm 0.70$ & $1.65 \pm 1.16$ \\
\hline
\end{tabular}

Rat pups were subjected to either i.c. application of $\mathrm{NaCl}(n=17)$ or LPS $(n=19)$. One hour later, the animals underwent unilateral carotid artery occlusion followed by 60 min of hypoxia (HI). Score 1, 0-5\% damage; score 2, 5-50\% damage; score 3, 50-95\% damage; score 4, 95-99\% damage; score $5,100 \%$ damage. Values are given as means \pm SD. Statistically significant differences between control and study group were evaluated by Mann-Whitney $U$ test $(* p<0.05, * * p<0.01, * * * p<0.001)$.

brain damage in neonatal rats. In most studies on LPS and ischemic neuronal injury, preconditioning effects were described with reduced brain injury when LPS was applied several days before middle cerebral artery occlusion in adult rats (28). However, the time interval between the primary and secondary insults seems to be the most important variable determining whether an alleviation or an aggravation of the final outcome is observed. Thus, sensitization of the immature brain to injury has been demonstrated after repeated brief episodes of cerebral hypoxia-ischemia (26). A similar relationship may exist between LPS and hypoxia-ischemia.

The mechanisms that mediate the sensitizing effects of LPS to hypoxic-ischemic insults are little understood. In a recent study on neonatal rats, altered expression of CD14 mRNA and toll-like receptor-4 mRNA (TLR) were detected in the brain after i.p. injection of LPS and a subsequent hypoxic-ischemic insult (14). After binding LPS, the membrane-bound and soluble forms of CD14 promote the activation of myeloid (monocyte/macrophage/polymorphonuclear leukocyte) and nonmyeloid (endothelial/epithelial) cells, respectively (29). The elusive signaling unit of the LPS receptor includes members of the human TLR family. The cytoplasmic tail of TLR has a similar sequence homology to the IL-1 receptor I, and TLR stimulation induces synthesis of proinflammatory cytokines, such as IL-1 and TNF- $\alpha$ (30; for review 31). IL-1 mediates ischemic, excitotoxic, and traumatic brain injury, probably through multiple actions on glia, neurons, and the vasculature. TNF- $\alpha$ has also been reported to contribute to neuronal injury (32). Altered expression of CD14 and TLR-4 may therefore be one of the underlying mechanisms through which LPS sensitizes the immature brain to a subsequent hypoxic-ischemic insult (14).

It is interesting to note that in the present study, neuronal cell damage was aggravated only in the cortex but not in deeper brain structures such as the striatum and the hippocampus. We
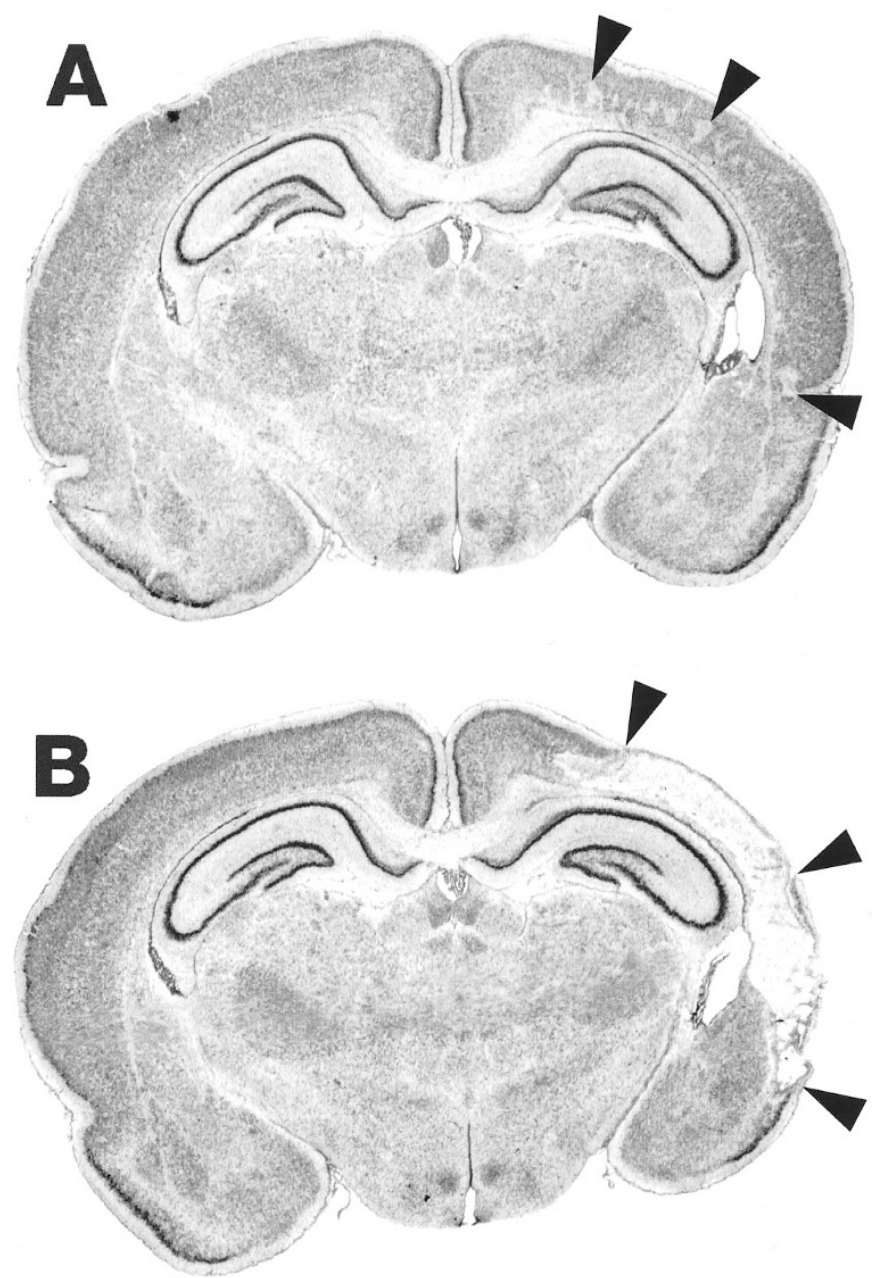

Figure 1. Photomicrographs showing coronal sections (A $2.0 \mathrm{~mm}$ ) of $\mathrm{NaCl}-$ treated $(A)$ and LPS-treated $(B)$ rat pups that were subjected to $60 \mathrm{~min}$ of hypoxia-ischemia. Sections were stained with cresyl-violet/fuchsin. Whereas only selective neuronal necrosis (arrowheads) could be observed in the animals that received $\mathrm{NaCl}$, the i.c. application of LPS sensitized the immature brain to a subsequent hypoxic-ischemic insult and resulted in cerebral infarction (arrowheads).

speculate that the TNF- $\alpha$ released in the leptomeninges penetrated the underlying cerebral cortex and accelerated cyclooxygenase 2 and the inducible form of nitric oxide synthase in the microglia (33). Radicals produced through these pathways are widely known to injure the neuronal network. In addition, TNF- $\alpha$ has been reported to activate the Fas-receptor system and to cause subsequent apoptotic cell death (for review 31). Another mechanism by which TNF- $\alpha$ could injure the immature brain is its stimulatory effects on metalloproteinases. These enzymes are known to degrade virtually all components of the extracellular matrix. They also lyse the subendothelial basement membrane, which forms the blood-brain barrier around cerebral capillaries and mediates the extravasation of leukocytes $(27,34)$. However, additional experiments will have to be performed to clarify whether these mechanisms are involved in the LPS-induced sensitization of the immature brain to ischemia.

Recently, Eklind et al. (14) also described a sensitization of the immature rat brain to hypoxic-ischemic insults by i.p. 


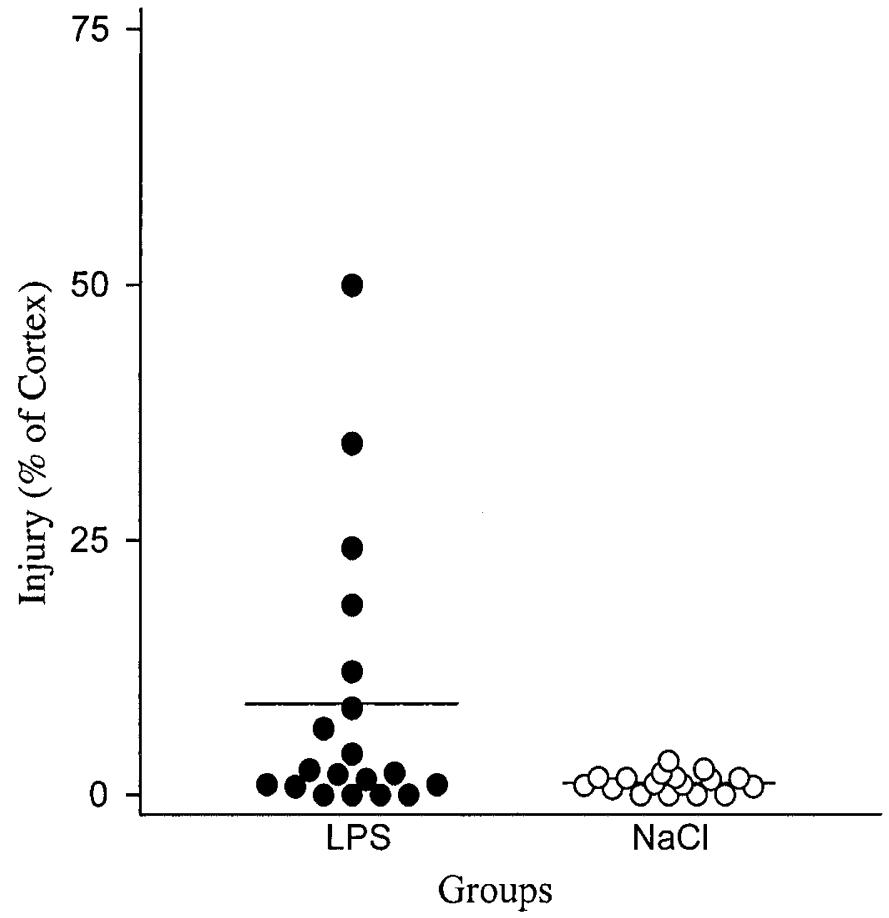

Figure 2. Effect of LPS (5 $\mu \mathrm{g} / \mathrm{animal}$ intracisternally) on subsequent hypoxic-ischemic brain damage in neonatal rats. Neuronal injury to the cortex was defined as areas of decreased neuronal density, confirmed by simultaneous bright field microscopy, and the area of cortical brain damage was expressed as percentage of the total cortex in each section. Statistically significant differences between control and study group were evaluated by unpaired $t$ test $(* p<0.03)$. injection of LPS $(0.3 \mathrm{mg} / \mathrm{kg}$ body $\mathrm{wt})$. The aggravation of the hypoxic-ischemic insult that they observed was more pronounced than that found in the present study. There could be various reasons for this: the LPS have been taken from different strains of bacteria and have been applied via differing routes in the two studies. Furthermore, the studies vary with regard to end points investigated. Whereas the rat pups were killed $3 \mathrm{~d}$ after the insult in the former report, in the present investigation animals were not perfusion-fixed until the seventh day after hypoxia. Eklind et al. (14) also measured cerebral blood flow immediately after the insult using the antipyrine technique and did not detect any differences between their study and control groups. From these results, they concluded that the effects of LPS on the immature brain were not mediated by an alteration of cerebral vasculature. However, as shown in a recent study on fetal sheep, i.v. application of LPS at a much lower concentration $(50 \mu \mathrm{g} / \mathrm{kg}$ body wt) severely compromised fetal cardiovascular control and nearly arrested cerebral oxygen delivery shortly after superimposed hypoxia (15). LPS-induced effects on cerebral blood flow therefore cannot be excluded by a single measurement at the end of a hypoxic-ischemic insult. In the present study, LPS was injected directly into the cerebrospinal fluid. Severe disturbances of the cardiovascular system are unlikely under such conditions but cannot be excluded, because blood flow measurements were not performed. Some reports describe an increase in cerebral blood flow within the first hours after intracerebroventricular injection of LPS $(19,20)$. Although
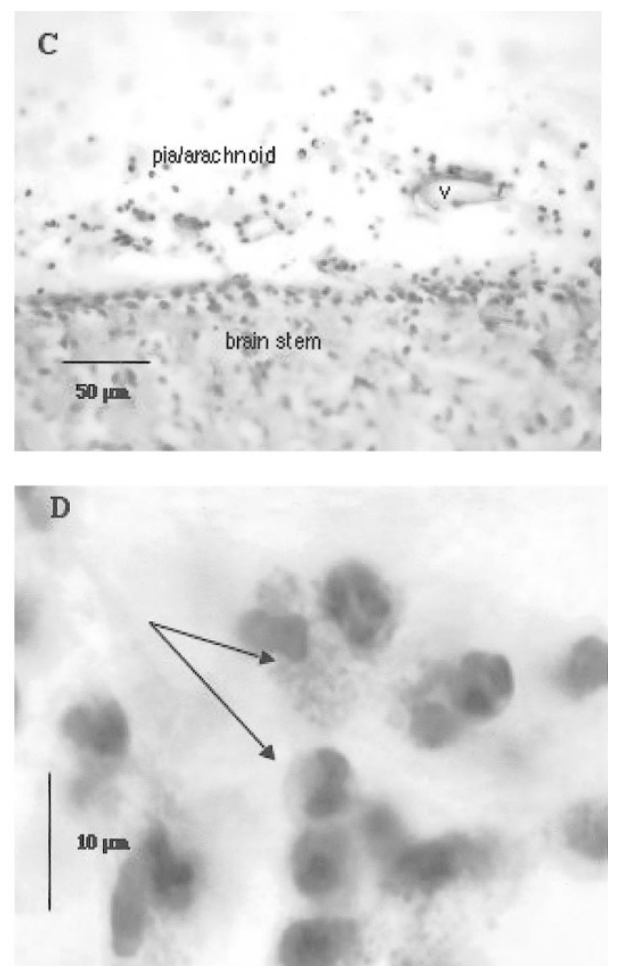

Figure 3. Representative photomicrographs of immunostaining for TNF- $\alpha$ in neonatal rat brain-stem sections. Anti-rat TNF- $\alpha$ against TNF- $\alpha$ was used as primary antibody at a working dilution of 1:1000. After incubation with primary antibody at room temperature for $2 \mathrm{~h}$, the avidin-biotin-horseradish peroxidase system was used for detection of immunopositive cells. Rat pups were subjected to either i.c. application of $\mathrm{NaCl}(C)$ or LPS $(A)$. The immunoreactive product could be detected only in the leptomeninges of the animals of the study group. Bar $=50 \mu \mathrm{m}$. B, The cytoplasmic TNF- $\alpha$ staining is indicated by arrows. Preadsorption of the TNF- $\alpha$ antibody with the homologous antigen is shown in $D$. Bar $=10 \mu \mathrm{m}$. v, venous vessel. 
such changes in cerebral perfusion are very unlikely to exacerbate hypoxic-ischemic brain injury, LPS-induced alterations of cerebral blood flow that enhances the susceptibility of the immature brain to subsequent insults cannot be excluded in the present study.

Despite extensive histologic examination, we did not detect any white-matter injury in the brains investigated. This seems to contradict epidemiologic data reporting an association between inflammation and white matter damage in immature fetuses $(35,36)$. However, one should take into consideration that the endotoxin was applied intracisternally in the present study and that the expression of TNF- $\alpha$ could be observed only in the leptomeninges. It is therefore conceivable that the concentration of the administered LPS did not reach concentrations in the cerebral white matter high enough to activate the pathophysiological cascade leading to cellular damage. The same may be true for the striatum and the hippocampus. Because we did not perform immunohistochemistry to characterize the various cellular subtypes of the brain parenchyma, subtle injury to smaller groups of cells such as oligodendrocytes and their precursors cannot be fully excluded.

Expression of TNF- $\alpha$ could not be observed in the present study until $10 \mathrm{~h}$ after the application of LPS, which is somewhat later than in previous studies performed in adult mice (37). As already mentioned, differences in the bacterial strain from which the LPS was derived as well as differences in the maturity of the immunologic system of the studied animals may be responsible for this finding.

\section{CONCLUSION}

From the results of the present study, we conclude that i.c. application of LPS enhances the susceptibility to subsequent hypoxic ischemic brain damage in neonatal rats. The underlying mechanisms are not yet fully understood.

\section{REFERENCES}

1. Berger R, Garnier Y 1999 Pathophysiology of perinatal brain damage. Brain Res Brain Res Rev 30:107-134

2. Volpe JJ 1995 Neurology of the Newborn. Saunders, Philadelphia

3. Dammann O, Leviton A 1997 Maternal intrauterine infection, cytokines, and brain damage in the preterm infant. Pediatr Res 42:1-8

4. Nisswander KR, Gordon M 1972 The Women and Their Pregnancies. The Collaborative Perinatal Study of the National Institute of Neurological Diseases and Stroke. WB Saunders, Philadelphia, pp 252-256

5. Berger R, Bender S, Sefkow S, Klingmüller V, Künzel W, Jensen A 1997 Peri/ intraventricular haemorrhage: a cranial ultrasound study on 5286 neonates. Eur J Obstet Gynecol Reprod Biol 75:191-203

6. Paneth N, Pinto-Martin J, Gardiner J, Wallenstein S, Katsikiotis V, Hegyi T, Hiatt IM, Susser M 1993 Incidence and timing of germinal matrix/ventricular hemorrhage in low birth weight infants. Am J Epidemiol 137:1167-1176

7. Gatti S, Bartfai T 1993 Induction of tumor necrosis factor-alpha mRNA in the brain after peripheral endotoxin treatment: comparison with interleukin-1 family and interleukin-6. Brain Res 624:291-294

8. Hillhouse EW, Mosley K 1993 Peripheral endotoxin induces hypothalamic immunoreactive interleukin-1 beta in the rat. Br J Pharmacol 109:289-290

9. Van Dam AM, Bauer J, Tilders FJ, Berkenbosch F 1995 Endotoxin-induced appearance of immunoreactive interleukin-1 beta in ramified microglia in rat brain: a light and electron microscopic study. Neuroscience 65:815-826
10. Barone FC, Arvin B, White RF, Miller A, Webb CL, Willette RN, Lysko PG, Feuerstein GZ 1997 Tumor necrosis factor-alpha. A mediator of focal ischemic brain injury. Stroke 28:1233-1244

11. Cai Z, Pan Z, Pang Y, Evans OB, Rhodes PG 2000 Cytokine induction in fetal rat brains and brain injury in neonatal rats after maternal lipopolysaccharide administration. Pediatr Res 47:64-72

12. Yoon BH, Kom CJ, Romero R, Jun JK, Park KH, Choi ST, Chi JG 1997 Experimentally induced intrauterine infection causes fetal brain white matter lesions in rabbits. Am J Obstet Gynecol 177:797-802

13. Gilles FH, Averill DR Jr, Kerr CS 1977 Neonatal endotoxin encephalopathy. Ann Neurol 2:49-56

14. Eklind S, Mallard C, Leverin A, Gilland E, Blomgren K, Mattsby-Baltzer I, Hagberg H 2001 Bacterial endotoxin sensitizes the immature brain to hypoxic-ischaemic injury. Eur J Neurosci 13:1101-1106

15. Garnier Y, Coumans ABC, Berger R, Jensen A, Hasaart THM 2001 Endotoxemia severely affects circulation during normoxia and asphyxia in immature fetal sheep. J Soc Gynecol Investig 8:134-142

16. Nelson KB, Grether JK 1998 Potentially asphyxiating conditions and spastic cerebral palsy in infants of normal birth weight. Am J Obstet Gynecol 179:507-513

17. Leib SL, Clements JM, Lindberg RL, Heimgartner C, Loeffler JM, Pfister LA, Tauber MG, Leppert D 2001 Inhibition of matrix metalloproteinases and tumour necrosis factor alpha converting enzyme as adjuvant therapy in pneumococcal meningitis. Brain 124:1734-1742

18. Rice JED, Vannucci RC, Brierley JB 1981 The influence of immaturity on hypoxicischemic brain damage in the rat. Ann Neurol 9:131-141

19. Okamoto H, Ito O, Roman RJ, Hudetz AG 1998 Role of inducible nitric oxide synthase and cyclooxygenase-2 in endotoxin-induced cerebral hyperemia. Stroke 29:1209-1218

20. Brian JE, Heistad DD, Faraci FM 1995 Dilatation of cerebral arterioles in response to lipopolysaccharide in vivo. Stroke 26:277-281

21. Bona E, Johansson BB, Hagberg H 1997 Sensorimotor function and neuropathology five to six weeks after hypoxia-ischemia in seven-day-old rats. Pediatr Res 42:678683

22. Sherwood NM, Timiras PS 1970 A Stereotaxic Atlas of the Developing Rat Brain. University of California Press, Berkeley, pp 15-75

23. Brown AW, Brierley JB 1972 Anoxic-ischaemic cell change in rat brain light microscopic and fine structural observations. J Neurol Sci 16:59-84

24. Berger R, Lehmann T, Karcher J, Garnier Y, Jensen A 1998 Low dose flunarizine protects the fetal brain from ischemic injury in sheep. Pediatr Res 44:277-282

25. Berger R, Lehmann T, Karcher J, Schachenmayr W, Jensen A 1996 Relation between cerebral oxygen delivery and neuronal cell damage in fetal sheep near term. Reprod Fertil Dev 8:317-321

26. Mallard EC, Williams CE, Gunn AJ, Gunning MI, Gluckman PD 1993 Frequent episodes of brief ischemia sensitize the fetal sheep brain to neuronal loss and induce striatal injury. Pediatr Res 33:61-65

27. Leib SL, Leppert D, Clements J, Täuber MG 2000 Matrix metalloproteinases contribute to brain damage in experimental pneumococcal meningitis. Infect Immun 68:615-620

28. Dawson DA, Furuya K, Gotoh J, Nakao Y, Hallenbeck JM 1999 Cerebrovascular hemodynamics and ischemic tolerance: lipopolysaccharide-induced resistance to focal cerebral ischemia is not due to changes in severity of the initial ischemic insult, but is associated with preservation of microvascular perfusion. J Cereb Blood Flow Metab 19:616-623

29. Ulevitch RJ, Tobias PS 1995 Receptor-dependent mechanisms of cell stimulation by bacterial endotoxin. Annu Rev Immunol 13:437-457

30. Kopp EB, Medzhitov R 1999 The Toll-receptor family and control of innate immunity. Curr Opin Immunol 11:13-18

31. Ulevitch RJ, Tobias PS 1999 Recognition of gram-negative bacteria and endotoxin by the innate immune system. Curr Opin Immunol 11:19-22

32. Allan SM, Rothwell NJ 2001 Cytokines and acute neurodegeneration. Nat Rev Neurosci 2:734-744

33. Serou MJ, DeCoster MA, Bazan NG 1999 Interleukin-1 beta activates expression of cyclooxygenase- 2 and inducible nitric oxide synthase in primary hippocampal neuronal culture: platelet-activating factor as a preferential mediator of cyclooxygenase-2 expression. J Neurosci Res 58:593-598

34. Rosenberg GA, Estrada EY, Dencoff JE, Stetler-Stevenson WG 1995 Tumor necrosis factor-alpha-induced gelatinase B causes delayed opening of the blood-brain barrier: an expanded therapeutic window. Brain Res 703:151-155

35. Leviton A, Paneth N, Reuss ML, Susser M, Allred EN, Dammann O, Kuban K, Van Marter LJ, Pagano M, Hegyi T, Hiatt M, Sanocka U, Shahrivar F, Abiri M, Disalvo D, Doubilet P, Kairam R, Kazam E, Kirpekar M, Rosenfeld D, Schonfeld S, Share J, Collins M, Genest D, Shen-Schwarz S 1999 Maternal infection, fetal inflammatory response, and brain damage in very low birth weight infants. Developmental Epidemiology Network Investigators. Pediatr Res 46:566-575

36. Wu YW, Colford JM Jr 2000 Chorioamnionitis as a risk factor for cerebral palsy: a meta-analysis. JAMA 284:1417-1424

37. Faggioni R, Fantuzzi G, Villa P, Buurman W, van Tits LJ, Ghezzi P 1995 Independent down-regulation of central and peripheral tumor necrosis factor production as a result of lipopolysaccharide tolerance in mice. Infect Immun 63:1473-1477 